PROCEEDINGS OF THE

AMERICAN MATHEMATICAL SOCIETY

Volume 128, Number 9, Pages 2577-2583

S 0002-9939(00)05319-3

Article electronically published on February 29, 2000

\title{
A SHARP ESTIMATE FOR EXTREMAL FUNCTIONS
}

\author{
KEHE ZHU
}

(Communicated by Albert Baernstein II)

\begin{abstract}
We prove a sharp pointwise estimate for extremal functions of invariant subspaces of some weighted Bergman spaces on the unit disk. The allowed weights include standard radial weights and logarithmically subharmonic weights.
\end{abstract}

\section{INTRODUCTION}

Let $\mathbb{D}$ be the unit disk in the complex plane $\mathbb{C}$ and let $\omega$ be a nonnegative weight function in $\mathbb{D}$. Throughout the paper we further assume that $\omega$ is continuous and has isolated zeros in $\mathbb{D}$.

For $0<p<\infty$ let $A_{\omega}^{p}$ denote the closure of the polynomials in the space $L^{p}(\mathbb{D}, \omega d A)$, where $d A$ is the normalized area measure on $\mathbb{D}$. The spaces $A_{\omega}^{p}$ will be called weighted Bergman spaces.

Under our assumptions on the weight function it is easy to show that each point evaluation in $\mathbb{D}$ is a bounded linear functional on each $A_{\omega}^{p}$. In particular $A_{\omega}^{2}$ is a reproducing Hilbert space. Let $K^{\omega}$ denote the reproducing kernel of $A_{\omega}^{2}$. See [2] and [6] for general properties of such reproducing kernels.

We call a closed subspace $I$ of $A_{\omega}^{p}$ invariant if $p f \in I$ whenever $f \in I$ and $p$ is a polynomial. For an invariant subspace $I$ of $A_{\omega}^{p}$ we let $n_{I}$ denote the smallest nonnegative integer $n$ such that there exists $f \in I$ with $f^{(n)}(0) \neq 0$ and consider the extremal problem

$$
\sup \left\{\operatorname{Re} f^{(n)}(0): f \in I,\|f\|_{p} \leq 1\right\},
$$

where $n=n_{I}$. Any solution to such a problem will be called an extremal function in $A_{\omega}^{p}$.

The purpose of this paper is to prove the following sharp estimate for extremal functions in weighted Bergman spaces for a large class of weights.

Theorem. Suppose $\log \omega$ is subharmonic. Then for all $0<p<\infty$ and all extremal functions $G$ in $A_{\omega}^{p}$ we have

$$
|G(z)|^{p} \leq\left(1-|z|^{2}\right) K^{\omega}(z, z)
$$

for all $z \in \mathbb{D}$.

It is customary to call $\omega$ logarithmically subharmonic when $\log \omega$ is subharmonic. The theorem above will be proved under the assumption that $\omega$ is logarithmically

Received by the editors July 20, 1998 and, in revised form, October 7, 1998.

1991 Mathematics Subject Classification. Primary 30C40, 46E22, 47A45.

(C) 2000 American Mathematical Society 
subharmonic. However, we will explain that the result actually holds in many other situations as well, including the case of standard radial weights.

This paper is an expanded version of my previously circulated manuscript " $A$ growth estimate for extremal functions". I thank the referee for carefully suggesting the revision.

\section{Estimate for a general funCtion in $A_{\omega}^{p}$}

In this section we give a sharp pointwise estimate for functions in $A_{\omega}^{p}$. The unweighted case of our result can be found in 10.

A weight function $\omega$ will be called representing at 0 if

$$
\int_{\mathbb{D}} h(z) \omega(z) d A(z)=h(0)
$$

for all bounded harmonic functions $h$ in $\mathbb{D}$.

Lemma 1. Suppose $\omega$ is logarithmically subharmonic and representing at 0 . Then

$$
\int_{\mathbb{D}}|f(z)|^{p} \omega(z) d A(z) \geq \int_{\mathbb{D}}|f(z)|^{p} d A(z) \geq|f(0)|^{p}
$$

for all $0<p<\infty$ and $f \in A_{\omega}^{p}$.

Proof. The second inequality is trivial, and the first one follows from the main integral formula in [4]. See also the proof of Proposition 2.5 in [1].

For any $z \in \mathbb{D}$ we let $k_{z}^{\omega}$ denote the normalized reproducing kernel of $A_{\omega}^{2}$, namely,

$$
k_{z}^{\omega}(w)=\frac{K^{\omega}(w, z)}{\sqrt{K^{\omega}(z, z)}}, \quad w \in \mathbb{D}
$$

Writing

$$
\left|k_{z}^{\omega}\right|^{2}=k_{z}^{\omega} \overline{k_{z}^{\omega}}
$$

and applying the reproducing property of the kernel function $K^{\omega}$, we easily arrive at the reproducing formula

$$
f(z)=\int_{\mathbb{D}} f(w)\left|k_{z}^{\omega}(w)\right|^{2} \omega(w) d A(w)
$$

where $f$ is any bounded analytic function in $\mathbb{D}$. Since $\left|k_{z}^{\omega}(w)\right|^{2} \omega(w) d A(w)$ is a probability measure, an application of Hölder's inequality yields

$$
|f(z)|^{p} \leq \int_{\mathbb{D}}|f(w)|^{p}\left|k_{z}^{\omega}(w)\right|^{2} \omega(w) d A(w)
$$

for all $p \geq 1$ and all analytic functions $f$ (first do this for bounded analytic functions and then use a limit argument for the general case). We now show that the inequality above also holds for $0<p<1$.

Lemma 2. Suppose $\omega$ is logarithmically subharmonic. Then for all $0<p<\infty$ and all $f$ in $A_{\omega}^{p}$ we have

$$
|f(z)|^{p} \leq \int_{\mathbb{D}}|f(w)|^{p}\left|k_{z}^{\omega}(w)\right|^{2} \omega(w) d A(w)
$$

for all $z \in \mathbb{D}$. 
Proof. Without loss of generality we may assume $f$ is bounded.

Fix $z \in \mathbb{D}$ and let $\varphi_{z}$ be the Möbius map defined by

$$
\varphi_{z}(w)=\frac{z-w}{1-\bar{z} w}, \quad w \in \mathbb{D} .
$$

Then a change of variables leads to

$$
\int_{\mathbb{D}}|f(w)|^{p}\left|k_{z}^{\omega}(w)\right|^{2} \omega(w) d A(w)=\int_{\mathbb{D}}\left|f \circ \varphi_{z}(w)\right|^{p} \lambda(w) d A(w),
$$

where

$$
\lambda(w)=\left|k_{z}^{\omega} \circ \varphi_{z}(w)\right|^{2} \omega \circ \varphi_{z}(w) \frac{\left(1-|z|^{2}\right)^{2}}{|1-\bar{z} w|^{4}} .
$$

It is easy to check that $\lambda$ is representing at 0 . Also, $\lambda$ is logarithmically subharmonic since $\omega$ is. Applying Lemma 1 with $\omega$ replaced by $\lambda$, we obtain

$$
\int_{\mathbb{D}}|f(w)|^{p}\left|k_{z}^{\omega}(w)\right|^{2} \omega(w) d A(w) \geq\left|f \circ \varphi_{z}(0)\right|^{p}=|f(z)|^{p}
$$

for all $z \in \mathbb{D}$.

We can now prove the main result of the section.

Theorem 3. Suppose $\omega$ is logarithmically subharmonic. If $0<p<\infty$ and $f$ is a unit vector in $A_{\omega}^{p}$, then

$$
|f(z)|^{p} \leq K^{\omega}(z, z)
$$

for all $z \in \mathbb{D}$.

Proof. By 2] each $k_{z}^{\omega}$ is nonvanishing. Thus we can write

$$
1=\int_{\mathbb{D}}|f(w)|^{p} \omega(w) d A(z)=\int_{\mathbb{D}}\left|f(w) k_{z}^{\omega}(w)^{-\frac{2}{p}}\right|^{p}\left|k_{z}^{\omega}(w)\right|^{2} \omega(w) d A(w) .
$$

An application of Lemma 2 then yields

$$
\left|f(z) k_{z}^{\omega}(z)^{-\frac{2}{p}}\right|^{p} \leq 1,
$$

or

$$
|f(z)|^{p} \leq K^{\omega}(z, z)
$$

for all $z \in \mathbb{D}$.

\section{Estimate FOR EXtREmal FUnCTIONS}

In this section we show that extremal functions in $A_{\omega}^{p}$ grow more slowly near the unit circle than a typical function in $A_{\omega}^{p}$, and we will see exactly how much more slowly.

Lemma 4. Let $0<p<\infty$ and let $G$ be an extremal function in $A_{\omega}^{p}$. Then

$$
\int_{\mathbb{D}}|G(z)|^{p} h(z) \omega(z) d A(z)=h(0)
$$

for all bounded harmonic functions $h$ in $\mathbb{D}$. 
Proof. This follows from the same variational arguments used in [3] and [4]. In fact, comparing the function $G$ with

$$
g(z)=\frac{G(z)+a z^{k} G(z)}{\left\|G+a z^{k} G\right\|}
$$

where $k \geq 1$ and $a \in \mathbb{C}$, leads to

$$
\int_{\mathbb{D}}|G(z)|^{p}\left|1+a z^{k}\right|^{p} \omega(z) d A(z) \geq 1 .
$$

Fix any real $\theta$ and let $a=t e^{i \theta}$, where $t \in(-\infty, \infty)$. Then the differentiable function

$$
f(t)=\int_{\mathbb{D}}|G(z)|^{p}\left|1+t e^{i \theta} z^{k}\right|^{p} \omega(z) d A(z)
$$

achieves its minimum at $t=0$, so that $f^{\prime}(0)=0$. A simple computation then gives

$$
\operatorname{Re} e^{i \theta} \int_{\mathbb{D}}|G(z)|^{p} z^{k} \omega(z) d A(z)=0 .
$$

Since $\theta$ is arbitrary, we must have

$$
\int_{\mathbb{D}}|G(z)|^{p} z^{k} \omega(z) d A(z)=0
$$

for all $k \geq 1$, which easily implies that

$$
\int_{\mathbb{D}}|G(z)|^{p} h(z) \omega(z) d A(z)=h(0)
$$

for all bounded harmonic functions.

Corollary 5. If $G$ is an extremal function in $A_{\omega}^{p}$, where $0<p<\infty$, then

$$
\int_{\mathbb{D}}|G(z)|^{p} h(z) \omega(z) d A(z) \leq h(0)
$$

for all nonnegative harmonic functions.

Proof. For $0<r<1$ define $h_{r}$ by $h_{r}(z)=h(r z), z \in \mathbb{D}$. The desired result then follows from the lemma above and Fatou's lemma.

For $0<p<\infty$ let $H^{p}$ denote the standard Hardy space consisting of analytic functions $f$ in $\mathbb{D}$ with

$$
\|f\|_{H^{p}}^{p}=\sup _{0<r<1} \frac{1}{2 \pi} \int_{0}^{2 \pi}\left|f\left(r e^{i t}\right)\right|^{p} d t<\infty .
$$

Lemma 6. If $0<p<\infty$ and $G$ is an extremal function in $A_{\omega}^{p}$, then $G$ is a contractive (pointwise) multiplier from the Hardy space $H^{p}$ into $A_{\omega}^{p}$.

Proof. Fix a function $f \in H^{p}$ and let $h$ be the least harmonic majorant of $|f|^{p}$, that is,

$$
h(z)=\frac{1}{2 \pi} \int_{0}^{2 \pi} \frac{1-|z|^{2}}{\left|z-e^{i t}\right|^{2}}\left|f\left(e^{i t}\right)\right|^{p} d t, \quad z \in \mathbb{D} .
$$

Using $|f|^{p} \leq h$ and applying Corollary 5, we obtain

$$
\int_{\mathbb{D}}|G(z) f(z)|^{p} \omega(z) d A(z) \leq h(0)=\|f\|_{H^{p}}^{p},
$$

so that $G$ is a contractive multiplier from $H^{p}$ into $A_{\omega}^{p}$.

We now arrive at the main result of the paper. 
Theorem 7. Suppose $\omega$ is logarithmically subharmonic. If $0<p<\infty$ and $G$ is an extremal function in $A_{\omega}^{p}$, then

$$
|G(z)|^{p} \leq\left(1-|z|^{2}\right) K^{\omega}(z, z)
$$

for all $z \in \mathbb{D}$.

Proof. Fix $z \in \mathbb{D}$ and let

$$
f(w)=\left(\frac{1-|z|^{2}}{(1-\bar{z} w)^{2}}\right)^{\frac{1}{p}}, \quad w \in \mathbb{D} .
$$

Then $f$ is a unit vector in $H^{p}$. By Lemma 6, the norm of $G f$ in $A_{\omega}^{p}$ does not exceed 1. According to Theorem [3, we must have

$$
|G(z) f(z)|^{p} \leq K^{\omega}(z, z),
$$

or

$$
|G(z)|^{p} \leq\left(1-|z|^{2}\right) K^{\omega}(z, z),
$$

completing the proof of the theorem.

\section{Further REMARKS}

Carefully examining the arguments used in the previous sections we realize that the key to the proof of the main result is the inequality

$$
|f(z)|^{p} \leq \int_{\mathbb{D}}|f(w)|^{p}\left|k_{z}^{\omega}(w)\right|^{2} \omega(w) d A(w),
$$

where $f$ is analytic, and the fact that the kernel function $K^{\omega}$ is nonvanishing. If $1 \leq p<\infty$, then as was remarked earlier, the above inequality holds in general. We suspect that the above inequality also holds for $0<p<1$ without the assumption that $\omega$ be logarithmically subharmonic. We illustrate this with an example.

For $-1<\alpha<\infty$ let

$$
\omega_{\alpha}(z)=(\alpha+1)\left(1-|z|^{2}\right)^{\alpha}, \quad z \in \mathbb{D} .
$$

The weight function $\omega_{w}$ is not logarithmically subharmonic unless $-1<\alpha \leq 0$, although it is representing at the origin. It is well known (see 11] for example) that the kernel function corresponding to $\omega_{\alpha}$ is

$$
K^{\alpha}(z, w)=\frac{1}{(1-z \bar{w})^{2+\alpha}} .
$$

Clearly, this function is nonvanishing.

If $h$ is a nonnegative subharmonic function in $\mathbb{D}$, then the use of polar coordinates yields

$$
h(0) \leq \int_{\mathbb{D}} h(z) \omega_{\alpha}(z) d A(z) .
$$

Replace $h$ by $h \circ \varphi_{z}$ and make a change of variables. We arrive at

$$
h(z) \leq \int_{\mathbb{D}} h(w)\left|k_{z}^{\alpha}(w)\right|^{2} \omega_{\alpha}(w) d A(w),
$$

where

$$
k_{z}^{\alpha}(w)=\frac{K^{\alpha}(w, z)}{\sqrt{K^{\alpha}(z, z)}}
$$


is the normalized reproducing kernel corresponding to the weight $\omega_{\alpha}$. Let $h=|f|^{p}$, where $f$ is analytic in $\mathbb{D}$ and $0<p<\infty$. Then $h$ is subharmonic and so

$$
|f(z)|^{p} \leq \int_{\mathbb{D}}|f(w)|^{p}\left|k_{z}^{\alpha}(w)\right|^{2} \omega_{\alpha}(w) d A(w),
$$

which is our key inequality. Thus we have proved the following

Theorem 8. If $0<p<\infty$ and $G$ is an extremal function in $A_{\omega_{\alpha}}^{p}$, then

$$
|G(z)|^{p} \leq \frac{1}{\left(1-|z|^{2}\right)^{1+\alpha}}
$$

for all $z \in \mathbb{D}$.

The above result was first proved in [5] in the case $p=2$ and $\alpha=0$. In the case $\alpha=0$ and $0<p<\infty$, this result was mentioned in [9], and a slightly weaker version was proved in $[8]$.

Our approach in the paper also allows generalizations to higher dimensions and other domains. We omit the details, since no application of this in higher dimensions has yet been found. In the case of the unit disk, we point out that the estimate in Theorem 8 plays a crucial role in the study of zero sets for Bergman spaces with standard radial weights (see [7]).

Finally we mention that the estimates in Theorems 7 and 8 are sharp. To see this, consider the unweighted Bergman space $A^{p}$ (corresponding to $\alpha=0$ in Theorem 8 ) and consider the extremal function $G$ for the invariant subspace generated by the singular inner function

$$
S(z)=\exp \left(-\frac{1+z}{1-z}\right)
$$

It was shown in 4 that

$$
G(z)=(1+p)^{-\frac{1}{p}} S(z)\left(1+\frac{p}{1-z}\right)^{\frac{2}{p}}
$$

so that

$$
|G(z)|^{p}=\frac{1}{1-|z|^{2}} \cdot \frac{|p+1-z|^{2}}{p+1} \cdot \frac{1-|z|^{2}}{|1-z|^{2}} \exp \left(-p \frac{1-|z|^{2}}{|1-z|^{2}}\right) .
$$

Letting $z$ approach the unit circle along the edges of a Stolz angle at the point 1 , we see that $|G(z)|^{p}$ achieves the maximum rate of growth.

\section{REFERENCES}

[1] A. Aleman, S. Richter, and C. Sundberg, Beurling's theorem for the Bergman space, Acta Math. 177 (1996), 275-310. MR 98a:46034

[2] P. Duren, D. Khavinson, and H. Shapiro, Extremal functions in invariant subspaces of Bergman spaces, Illinois J. Math. 40 (1996), 202-210. [MR 97h:30069]

[3] P. Duren, D. Khavinson, H. Shapiro, and C. Sundberg, Contractive zero-divisors in Bergman spaces, Pacific J. Math. 157 (1993), 37-56. MR 94c:30048

[4] P. Duren, D. Khavinson, H. Shapiro, and C. Sundberg, Invariant subspaces in Bergman spaces and the bi-harmonic equation, Michigan Math. J. 41 (1994), 247-259. MR 95e:46030

[5] H. Hedenmalm, A factorization theorem for square area-integrable functions, J. Reine Angew. Math. 442 (1991), 45-68. MR 93c:30053

[6] H. Hedenmalm, An off-diagonal estimate of Bergman kernels, preprint, 1998.

[7] H. Hedenmalm, B. Korenblum, and K. Zhu, Theory of Bergman Spaces, Springer-Verlag, New York, to appear. 
[8] D. Khavinson and H. Shapiro, Invariant subspaces in Bergman spaces and Hedenmalm's boundary value problem, Ark. Math. 32 (1994), 309-321. MR 95m:31003]

[9] C. Sundberg, Analytic continuability of Bergman inner functions, Michigan Math. J. 44 (1997), 399-407. MR 98h:46022

[10] D. Vukotić, A sharp estimate for $A^{p}$ functions, Proc. Amer. Math. Soc. 123 (1993), 753-756. MR 93d:46042

[11] K. Zhu, Operator Theory in Function Spaces, Marcel Dekker, New York, 1990. MR 92c:47031

Department of Mathematics, State University of New York, Albany, New York 12222

E-mail address: kzhu@math.albany.edu 